albeit in a different way, by modulating ryanodine receptors in blood vessels. If so, the absence of FKBP12.6 would lead to an increase in blood pressure, requiring the heart to pump more forcefully. And the predicted outcome of more forceful cardiac contraction in the face of lasting high blood pressure is enlargement of the heart. I ndeed, $X$ in et al. found that male, but not female, FKBP12.6-null mice had high blood pressure ${ }^{1}$. This is consistent with the idea that cardiac hypertrophy in the males is a secondary consequence of elevated blood pressure, and that oestrogen opposes the effects of alack of FKBP12.6on blood pressure.

There are other possibilities, however. The increases in intracellular calcium levels seen in FKBP12.6-deficient animals might lead directly to an increase in the activity of the calcium-dependent transcription factor NFAT. Alternatively, because FKBP12 and FKBP12.6 not only regulate calcium release through ryanodine receptors but are also important components of the calcineurin protein complex, which regulates NFAT activity, it is possible that disruption of FKBP12 or FKBP12.6 in the heart or blood vessels leads to the activation of NFAT through effects on calcineurin. The activation of NFAT by either of these two mechanisms might induce a gene programme that causes an increase in blood pressure or cardiac hypertrophy. It is conceivable that oestrogen decreases theexpression or activity of NFAT or its target genes in the blood vessels or hearts of females. Whatever the answer, an understanding of the molecular mechanismsat work will no doubt becrucial to comprehending gender-based differences in cardiovascular function.

M ark T. N elson and Gerald M. Herrera are in the

Department of Pharmacology, U niversity of

Vermont, Given M edical Building, Burlington,

Vermont 05405-0068, USA.

e-mail: mtnelson@zoo.uvm.edu

1. Xin, H.-B. et al. Nature 416, 334-337 (2002).

2. Shou, W. et al. Nature 391, 489-492 (1998).

3. Molkentin, J. D. et al. Cell 93, 215-228 (1998).

4. Graef, I. A., Chen, F., Chen, L., Kuo, A. \& Crabtree, G. R. Cell 105, 863-875 (2001).

5. Stevenson, A. S., Gomez, M. F., Hill-Eubanks, D. C. \& Nelson, M. T. J. Biol. Chem. 276, 15018-15024 (2001).

6. Marks, A. R. Physiol. Rev. 76, 631-649 (1996).

7. Mendelsohn, M. E. \& Karas, R. H. N. Engl. J. M ed. 340, 1801-1811 (1999).

8. Wellman, G. C., Bonev, A. D., Nelson, M. T. \& Brayden, J. E. Circ. Res. 79, 1024-1030 (1996)

9. Geary, G. G. et al. J. Appl. Physiol. 91, 2391-2399 (2001).

10.Zhu, Y. et al. Science 295, 505-508 (2002).

Materials science

\title{
Breaking the neural code
}

Adam Curtis

The precise information that is conveyed between nerve cells remains unknown. Networks of nerve cells grown on silicon chips, using a polyester as a guide, may bring us closer to translating the elusive neural language.

W ithin a nerve cell, or neuron, differences in electrical potential encode information, and messages can be passed on to other neurons through connections that may bechemical or electrical. But, like nineteenth-century linguists contemplating the hieroglyphs of the Rosetta Stone, wehave only vagueclues as to the symbolism and language, let alone the grammar or syntax, of neural messages. We will never digup aneurobiological RosettaStone- but we might be able to synthesize our own. In a notable paper in Advanced M aterials, M erz and Fromherz ${ }^{1}$ havetaken usat least onestage towardsthis goal, and their work brings with it thepromiseof further discoveries.

To decipher theneural code, theaim is to grow networks of neurons in culture, with each neuron connected to others through synapsesthat form attheends of theneuron's tendril-like extensions, or neurites. Weneed to reproduce the connectivity that is seen anatomically in nervous systems and which, when destroyed, leads to loss of correct function. We suspect that the message passing across a synapsecarries instructions for how the information should be processed and possibly also routed, and in many cases we know what the correct (or at least normal) input and output are. What we would like to test are the effects of different connectivities and in put messages on theneuronal output.

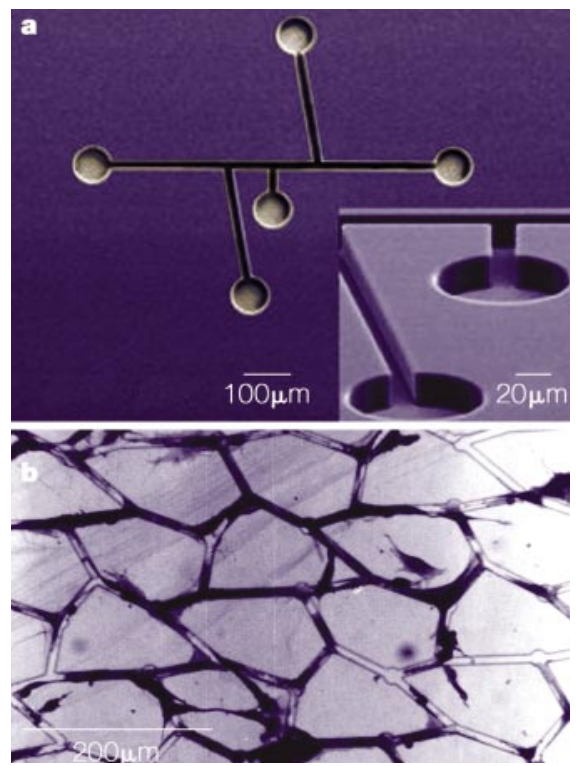

Work on growing neuronal networkshas been in progress for several years, although previous experiments paid insufficient attention to preventing the neurites from moving or becoming rearranged in the cultures; thiscan happen through themechanical forces generated by the cells themselves. But secure, long-term positioning of neurites (on a scale of days to weeks) has been achieved ${ }^{2}$, with lines of neurons immobilized using theprotein laminin. M aher et al. ${ }^{3}$ have also grown cells in deep pits, and recorded data from them. An alternative to network growing has also been tried. In an earlier, seminal experiment, Syed and collegues ${ }^{4}$ reconstructed neuronal circuits by delicate hand surgery and managed to rebuild a working circuit. But they did not explore modified circuits or the effects of changesin themessage.

Now M erz and Fromherz ${ }^{1}$ have built on the results of such experiments, and have obtained well-defined, if very small, stable networks of cultured neurons from the pond snail Lymnaea stagnalis. Using microfabrication techniques, they etched a series of pits and grooves in a polyester surfaceon asilicon substrate(Fig. 1a). Thedesign traps the cell bodies but allows neurites to grow along the grooves. Merz and Fromherz managed to identify pairs of nervecells that had formed connections and to record electrical signals from them. They did this by injecting current into one neuron and showing that a voltage change occurred in the neighbouring cell, indicating that electrical synapses had formed. M oreover, the electrical events observed in the networks were as expected, proving that the behaviour of theneurons was not being appreciably affected by the chemistry or structure of thepolyester-silicon growth material.

To record and stimulate electrical signals in their neuronal network, Merz and Fromherz used conventional intracellular

Figure 1 Artificially grown networks of neurons could be the key to deciphering the intricate code of neural messages. a, Merz and Fromherz ${ }^{1}$ etched pits and grooves in a polyester-silicon material to control the growth of neurites into a functioning network; pits $70-80 \mu \mathrm{m}$ in diameter match the size of the pond-snail neurons used in the experiment, and grooves up to $15 \mu \mathrm{m}$ wide and $30 \mu \mathrm{m}$ deep confine and guide the neurites into forming synaptic connections between neurons. b, Following a pattern suggested by Wilkinson ${ }^{6}$, Sandison et al. ${ }^{5}$ grew this neuronal network in grooves in silica - although the difficulty of confining the growth within the structure is apparent. 
glass microelectrodes, although their earlier work had used extracellular electrodes, based on field-effect transistors to amplify theweak electrical signals. Extracellular electrodes havean advantageover theintracellular kind becausetrappingneuronswith fixed electrodes, or piercing them with a microelectrode, can kill them. In fact, my own group has recently invented a manipulable extracellular electrode array ${ }^{5}$ that can be rapidly moved from siteto site. Theelectrode patterns can be matched to that of the network under test, although the array's extracellular position does reduce the magnitude of thesignal that can beextracted.

The growth technique of Merz and Fromherz, when combined with advances in electrode design, should open the way to investigating how connectivity and patterns of connection modify neural processing. In other words, it should be possible to test the consequences of specific changes in connectivity or in the electrically injected message on the output. Furthermore, as M erz and Fromherz $z^{1}$ point out, their system should allow the role of subthreshold signals to be assessed - these are events that do not lead directly to transmission of a neural message, but which may influence the electrical potentials of nervecells.

With the tools in place, the outstanding question ishow aneuronal network could be designed. Therearetwo sensibleapproaches: first, to try to mimic what is already found in nature; and second, to formally develop logically designed networks. Judith Wilkinson (quoted in ref. 6) has applied graph and tiling theory to design networks that should be able to propagate and compare messages (Fig. 1b). Theseareprobably theonlyin vitro systemsthatfeaturebranchingnetworksthat are just like those in real nervous systems. Such logically designed networks are just now being realized.

Despitethe progress reported by workers such as M erz and Fromherz, we should still proceed cautiously - for instance, wedo not even know whether there are (or aren't) several types of neural coding, or language, in a singleanimal. But, asin other code-breaking efforts, there will probably be a long period of data collection - revealing more and more of the neurobiological Rosetta Stone - until wehaveenough information to start interpreting theneural code.

Adam Curtis is at the Centre for Cell Engineering,

IBLS, Joseph Black Building, University of Glasgow, Glasgow G12 8Q Q, UK.

e-mail: a.curtis@bio.gla.ac.uk

1. Merz, M. \& Fromherz, P. Adv. M ater. 14, 141-144 (2002)

2. Lauer, L., Klein, C. \& Offenhauesser, A. Biomaterials 22, 1925-1932 (2001).

3. Maher, M. P. et al. Cell. Eng. 37, 110-118 (1999).

4. Syed, N. I., Bulloch, A. G. M. \& Lukowiak, K. Science 250, 282-285 (1990).

5. Sandison, M., Curtis, A. S. G. \& Wilkinson, C. D. W J. N eurosci. M ethods 114, 63-71 (2002)

6. Wilkinson, C. D. W. \& Curtis, A. S. G. PhysicsW orld 12, 45-48 (1999).

Immunology

\section{The Wright stuff}

Walter Gratzer

White cells in the bloodstream seek out and destroy invading bacteria. The explanation for the actual killing mechanism turns out to be wonderfully more subtle than previously thought.

O n page 291 of this issue, Segal and colleagues (Reeves et al.) ${ }^{1}$ cast new and unexpected light on one of the cornerstones of theimmunesystem. Their observations overturn the established wisdom that has guided the study of phagocytosis and pose questions about the role of reactive oxygen species in destroying invading pathogens.

It is 120 years since Elie M etchnikoff discovered phagocytes - cells that engulf and devour bacteria and other invaders of the bloodstream. This 'innate immunological response' is the body's first line of defence against such violations, and it depends predominantly on the most abundant class of phagocytic cells, the most numerous white cells in the blood, the neutrophils. Sufferers from the rare, hereditary chronic granulomatous disease (CGD), whose neutrophils are defective, die from bacterial and fungal infections if they are not treated. (M etchnikoff later came to believe that, with the passage of theyears, phagocytic cells become incontinent, rampage through our tissues, gnaw at our vitals and cause the catastrophe that werecognizeasageing.)

Neutrophils surge towards the site of an infection and engulf bacteria by a well-characterized process (opsonization) that requires blood serum proteins. They then destroy theinvoluntary guest trapped within a phagocytic vacuole. The cytoplasm of the neutrophil is rich in granules (whence its original description as a granulocyte), which, on activation, discharge their contentsinsidethephagocytic vacuole.

The picture of the killing process that has dominated thinking over the past 40 years is that the lethal agents are the highly reactive

\section{Quantum optics \\ Light corralled \\ Traditional optical experiments,} such as splitting rays of light into various colours with a prism, have had the attraction of being visible to the naked eye. Modern methods of confining light within microscopic structures, and tailoring its interaction with matter on atomic scales, are taking optics into the quantum realm. Making the results visible is not straightforward. But a beautiful example comes from C. Chicanne et al. (Physical Review Letters 88, 097402; 2002), who have designed a way of taking snapshots of intricate light interference patterns in tiny photonic structures. One of their snapshots is shown here.

The images are reminiscent of the 'quantum corrals' for electrons, first created by Donald Eigler and colleagues in 1993, and produced with the scanning tunnelling microscope (STM) This instrument probes surfaces with a sharp needle,

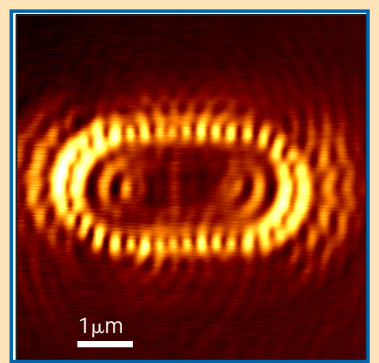

which can also be used to move loose atoms around on a metallic surface and position them into a closed loop. The electrons confined within these corrals interfere with one another and produce beautiful patterns, which the STM images make manifest. These experiments have been highly instructive for illustrating the quantum-mechanical principle that electrons can behave as waves.

Chicanne et al. now present an optical analogue of the quantum corral. Light has wave properties, of course, but interpreting the interference effects is not straightforward within a radius around a light source that is comparable to the wavelength of the light itself. To study this zone, Chicanne et al. made use of a relative of the STM, the scanning near-field optical microscope. Here, the surface probe is an optical fibre tapered to a sharp end that also illuminates the sample.

Much as in the experiments by Eigler and colleagues, Chicanne et al. first created corrals by carefully positioning particles of gold in a loop, in this example in the shape of a stadium. The optical corral is then imaged by scanning the fibre over the surface while collecting light transmitted though the transparent sample. The result is images of light interference patterns in a microscopic structure. The principles involved will help direct future observations, and tailored distribution, of light at atomic dimensions.

Liesbeth Venema 